AIP Appilied Physics

\title{
Energy and momentum relaxation dynamics of hot holes in modulation doped GalnNAs/GaAs quantum wells
}

Y. Sun and N. Balkan

Citation: J. Appl. Phys. 106, 073704 (2009); doi: 10.1063/1.3225997

View online: http://dx.doi.org/10.1063/1.3225997

View Table of Contents: http://jap.aip.org/resource/1/JAPIAU/v106/i7

Published by the American Institute of Physics.

\section{Related Articles}

Identification of the native defect doping mechanism in amorphous indium zinc oxide thin films studied using ultra high pressure oxidation

Appl. Phys. Lett. 102, 052101 (2013)

Detecting p-type conduction in Ba-doped InN

Appl. Phys. Lett. 102, 042109 (2013)

Chemical effect of $\mathrm{Si}+$ ions on the implantation-induced defects in $\mathrm{ZnO}$ studied by a slow positron beam

J. Appl. Phys. 113, 043506 (2013)

Influence of $\mathrm{Ga}$ doping on the $\mathrm{Cr}$ valence state and ferromagnetism in $\mathrm{Cr}$ : $\mathrm{ZnO}$ films

Appl. Phys. Lett. 102, 022414 (2013)

Doping incorporation paths in catalyst-free Be-doped GaAs nanowires

Appl. Phys. Lett. 102, 013117 (2013)

\section{Additional information on J. Appl. Phys.}

Journal Homepage: http://jap.aip.org/

Journal Information: http://jap.aip.org/about/about_the_journal

Top downloads: http://jap.aip.org/features/most_downloaded

Information for Authors: http://jap.aip.org/authors

\section{ADVERTISEMENT}

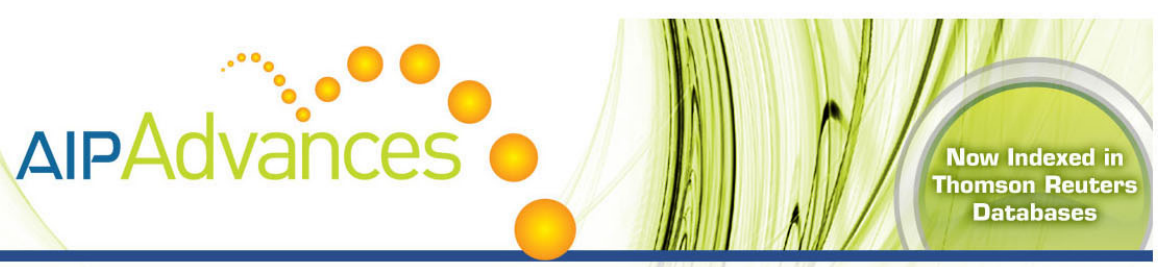

\section{Explore AIP's open access journal: •Rapid publication \\ - Article-level metrics \\ Post-publication rating and commenting}




\title{
Energy and momentum relaxation dynamics of hot holes in modulation doped GalnNAs/GaAs quantum wells
}

\author{
Y. Sun ${ }^{\text {a) }}$ and N. Balkan \\ School of Computer Science and Electronic Engineering, University of Essex, Wivenhoe Park, \\ Colchester CO4 3SQ, United Kingdom
}

(Received 5 May 2009; accepted 18 August 2009; published online 2 October 2009)

\begin{abstract}
We present the studies of energy and momentum relaxation dynamics of nonequilibrium holes in $\mathrm{Ga}_{x} \mathrm{In}_{1-x} \mathrm{~N}_{y} \mathrm{As}_{1-y} / \mathrm{GaAs}$ quantum well modulation doped with Be. Experimental results show that the real-space transfer (RST) of hot holes occurs via thermionic emission from the high-mobility GaInNAs quantum wells into the low-mobility GaAs barriers at a threshold electric field of $F$ $\sim 6 \mathrm{kV} / \mathrm{cm}$ at $T=13 \mathrm{~K}$. At this field the hole drift velocity saturates at $v_{d} \sim 1 \times 10^{7} \mathrm{~cm} / \mathrm{s}$. A slight increase in the field above the threshold leads to the impact ionization of acceptors in the barriers by the nonequilibrium holes. We observe and model theoretically a negative differential mobility effect induced by RST that occurs at an electric field of $F \sim 7 \mathrm{kV} / \mathrm{cm}$. The observed current surge at electric fields above $7 \mathrm{kV} / \mathrm{cm}$ is attributed to the hole multiplication induced by shallow impurity breakdown in the GaAs barrier and impact ionization in the high-field domain regime associated with the packet of RST of holes in the well. (C) 2009 American Institute of Physics.
\end{abstract}

[doi:10.1063/1.3225997]

\section{INTRODUCTION}

During the past decade, dilute nitrides, particularly the quaternary material system of GaInNAs/GaAs, have attracted a great deal of attention, both because of unusual physical properties and the potential applications for a variety of optoelectronic devices. ${ }^{1-5}$ The incorporation of a small amount of nitrogen $(<2 \%)$ into the host material has been shown to lead to large redshift of the band gap due to the large electronegativity of nitrogen and the possibility of lattice matching to GaAs substrate. ${ }^{6,7}$ A possible drawback of nitrogen incorporation appears to be the drastic drop in electron mobility as nitrogen is added. This is because nitrogen induces a strong perturbation to the conduction band of the matrix semiconductor leading to an enhancement of the electron effective mass. ${ }^{8-11}$ The mobility is further reduced by localized nitrogen related resonant scattering. ${ }^{12-15}$ However, the addition of $\mathrm{N}$ has negligible effect on the valence band; thus the hole mobility is not expected to be influenced by nitrogen incorporation. Therefore, in material with relatively high nitrogen incorporation, the hole mobility can be higher than the electron mobility at low temperature because of both smaller effective mass and the lack of scattering by nitrogen complexes as observed in our previous studies. ${ }^{16,17}$ The high hole mobility provides the motivation for the current work, i.e., to establish a full understanding of hole transport, particularly at high electric field for their potential application in ultrafast novel devices.

In $\mathrm{Ga}_{x} \mathrm{In}_{1-x} \mathrm{~N}_{y} \mathrm{As}_{1-y} / \mathrm{GaAs}$ quantum wells (QWs), hole confinement energy is much smaller than that for electrons, particularly at elevated nitrogen contents. Therefore, in $p$-modulation doped QWs, a moderately high electric field applied parallel to the layer interface may induce sufficient

\footnotetext{
${ }^{a)}$ Author to whom correspondence should be addressed. Electronic mail: ysunf@essex.ac.uk.
}

heating of holes for them to gain enough energy to overcome the small valence band discontinuity $(\sim 120 \mathrm{meV}$ in our calculation for the samples investigated in this work) and transfer in real space via thermal emission into the low-mobility $p$-doped GaAs layer. ${ }^{18}$ Therefore, a negative differential mobility (NDM) caused by real-space transfer (RST) of hot holes and accompanied by high-frequency space charge oscillations may be achieved similar to those predicted by Schöll and co-workers. ${ }^{19-22}$ The aim of the work presented here is to give the results of our studies on the longitudinal nonequilibrium hole transport in $p$-type modulation doped $\mathrm{Ga}_{1-x} \mathrm{In}_{x} \mathrm{~N}_{y} \mathrm{As}_{1-y} / \mathrm{GaAs}$ QWs and to explain the observed current-voltage characteristic using a simplified analytical model taking into account the effect of real-space hole transfer and the consequent impact ionization in both the barrier and QW.

\section{EXPERIMENT}

The layer structure of the sample used in the investigation is given in Fig. 1. The sample coded 1931 was grown by molecular beam epitaxy on a semi-insulating GaAs substrate and did not undergo rapid thermal annealing or slow thermal annealing. The mole fraction of indium and nitrogen in the $\mathrm{Ga}_{1-x} \mathrm{In}_{x} \mathrm{~N}_{y} \mathrm{As}_{1-y} \mathrm{QW}$ is $x=0.3$ and $y=0.015$, respectively. To establish the presence of the $\mathrm{Ga}_{1-x} \mathrm{In}_{x} \mathrm{~N}_{y} \mathrm{As}_{1-y}$ QWs, cw pho-

\begin{tabular}{|c|c|c|c|}
\hline Material & Thickness $(\AA)$ & Doping $\left(\mathrm{cm}^{-3}\right)$ & \\
\hline GaAs (Cap) & 500 & Be: $1 \times 10^{18}$ & \\
\hline GaAs (Barrier) & 200 & Be: $1 \times 10^{18}$ & \\
\hline GaAs (Spacer) & 50 & UD & \\
\hline $\mathrm{Ga}_{1-\mathrm{x}} \mathrm{In}_{\mathrm{x}} \mathrm{N}_{\mathrm{y}} \mathrm{As}_{1-\mathrm{y}} \mathrm{QW}$ & 70 & UD & \\
\hline GaAs(Spacer) & 50 & UD & $\mathrm{x} 3$ \\
\hline GaAs(Barrier) & 200 & Be: $1 \times 10^{18}$ & 1 \\
\hline GaAs (Buffer) & 500 & UD & $\downarrow$ \\
\hline
\end{tabular}

FIG. 1. The layer structures of the sample coded 1931. 

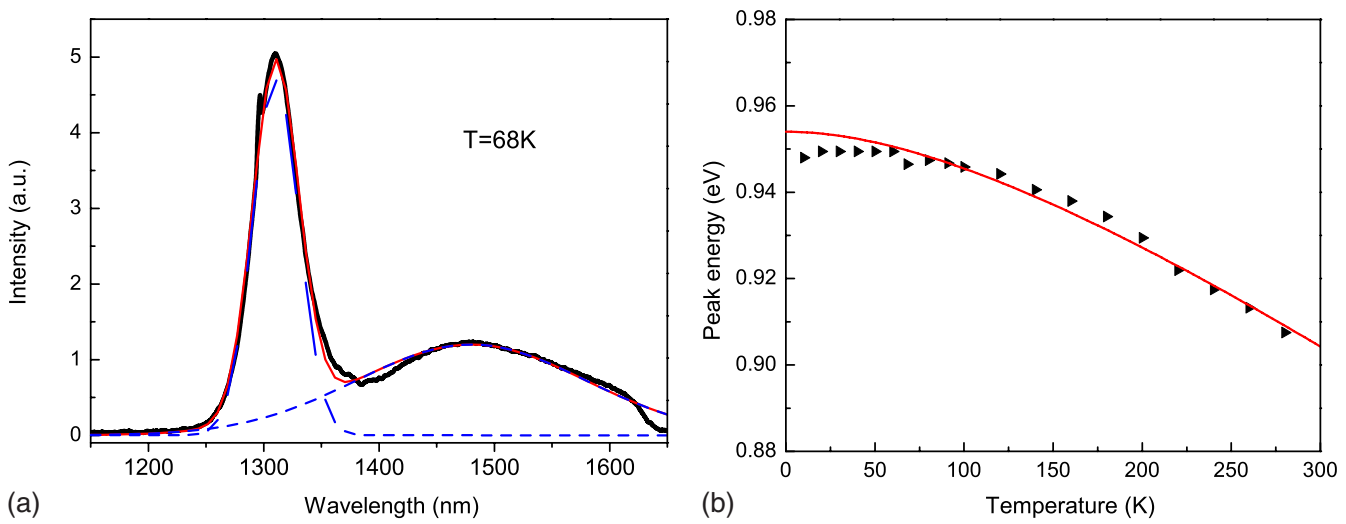

FIG. 2. (Color online) (a) The spectrum of sample 1931 measured at $68 \mathrm{~K}$ (solid line), overlaid with two fitted symmetrical Gaussian curves (thin broken line). (b) Temperature dependence of the QW peak emission energy.

toluminescence $(\mathrm{PL})$ spectra were measured as a function of temperature (these results were published elsewhere by us and will not be discussed here in detail). ${ }^{16}$ Here, for clarity we showed in Fig. 2(a) a typical PL spectrum measured at $T=68 \mathrm{~K}$. The spectrum reveals the presence of two overlapping components, a strong narrow QW emission (FWHM $\sim 27 \mathrm{meV}$ ) located at $0.95 \mathrm{eV}$ and a weak broad (FWHM $\sim 139 \mathrm{meV}) 0.8 \mathrm{eV}$ emission that may be associated with a transition from the arsenic interstitial-gallium vacancy $\left(\mathrm{As}_{i}-V_{\mathrm{Ga}}\right)$ defect center to the valence band in the $p$-doped GaAs layers as suggested by $\mathrm{Yu}$ and Shinohara. ${ }^{23-25}$

The PL emission is attributed to the recombination of free carriers as previously reported in modulation doped QWs; therefore, it has no S-shape temperature dependent behavior [Fig. 2(b)], which is commonly observed at low temperatures in the undoped material. ${ }^{26}$ The lack of the $S$ shape is primarily due to the high carrier density in modulation doped QWs screening efficiently the localized exciton transitions. Therefore, the observed behavior can reflect the true temperature dependence of band gap energy and be well fitted by an empirical relation between the band gap and temperature, as proposed by Polimeni et al. ${ }^{27}$ and Varshni, ${ }^{28}$ and previously reported by us. ${ }^{16,29}$

In order to determine the two-dimensional (2D) nature of the electrical conductivity, we employed the orthodox Hall measurements. The samples were fabricated in the form of Hall bars with channel lengths of $1.75 \mathrm{~mm}$ and widths of less than $0.2 \mathrm{~mm}$. Ohmic contacts were formed by alloying $\mathrm{Au}$ and $\mathrm{Au} / \mathrm{Zn}$. Measurements were recorded at lattice temperatures between 14 and $300 \mathrm{~K}$ at applied electric fields low enough to avoid any significant carrier heating effects. Indeed, both the Hall mobility and carrier density were independent of applied field within the measurement range. Figure 3 shows the Hall mobility and sheet carrier density plotted against the lattice temperature. The temperature behavior of the Hall density and mobility can be understood in terms of two parallel conducting channels, i.e., QWs and barriers where relative densities and mobilities change with temperature. ${ }^{30}$ The measured Hall carrier density and mobility are given by

$$
p_{H}=\frac{\left(p_{1} \mu_{1}+p_{2} \mu_{2}\right)^{2}}{p_{1} \mu_{1}^{2}+p_{2} \mu_{2}^{2}},
$$

$$
\mu_{H}=\frac{p_{1} \mu_{1}^{2}+p_{2} \mu_{2}^{2}}{p_{1} \mu_{1}+p_{2} \mu_{2}},
$$

where $p_{1} / \mu_{1}$ and $p_{2} / \mu_{2}$ are the hole densities/mobilities in the GaInNAs QWs and Be-doped GaAs barriers, respectively. $p_{1}$ will be constant if there is no thermal emission from the QWs, and $\mu_{1}, p_{2}$, and $\mu_{2}$ are all functions of temperature.

We see that at low temperatures, the free carrier density in the GaAs barrier (ionized acceptor) is negligible; therefore, the measured carrier density is constant and independent of temperature, $p_{H} \sim 1.2 \times 10^{12} \mathrm{~cm}^{-2}$, and corresponds to the hole density $p_{1}$ in the QWs. Therefore, the measured mobility, $\mu_{H} \sim 3125 \mathrm{~cm}^{-2} / \mathrm{V} \mathrm{s}$, represents the hole mobility, $\mu_{1}$, in the QWs. As the temperature increases, the unionized acceptors in the wide GaAs barriers fully ionize. Furthermore, with increasing temperature the free carriers in the wells acquire enough thermal energy to overcome the small potential barrier (hole confinement $\sim 110 \mathrm{meV}$ ) and spread into three-dimensional (3D) GaAs giving rise to the observed increasing carrier density. In Fig. 3, the mobility decreases slowly with increasing temperature up to $60 \mathrm{~K}$, where the mobility is mainly determined by remote impurity, interface roughness, and acoustic phonon scattering. ${ }^{31-33}$ At temperatures above $60 \mathrm{~K}$, the optical phonon scattering in the GaInNAs layers is the dominant process for momentum relax-

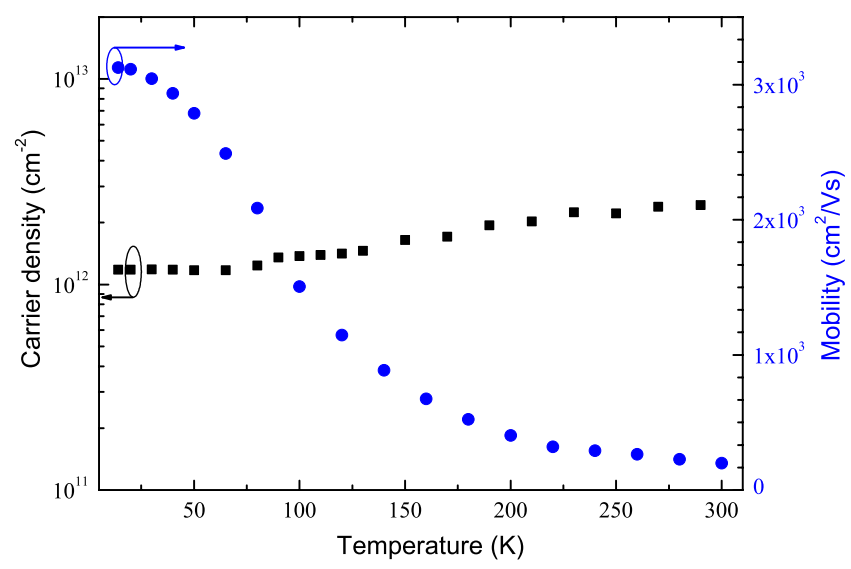

FIG. 3. (Color online) Temperature dependence of sheet carrier density (solid squares) and low-field Hall mobility (solid circles) of sample 1931. 


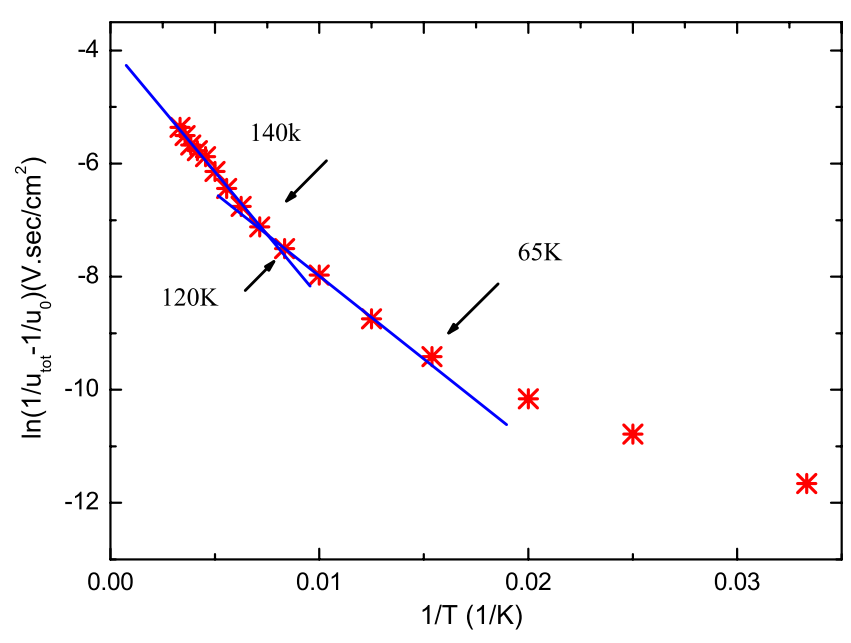

FIG. 4. (Color online) Fitting of $\ln \left(1 / \mu_{\text {tot }}-1 / \mu_{0}\right)$ vs inverse lattice temperature (line) which gives two LO phonon energies, 37 and $25 \mathrm{meV}$, respectively.

ation, resulting in a rapid decrease in $2 \mathrm{D}$ hole mobility. At temperatures above $120 \mathrm{~K}$, the Hall mobility increasingly corresponds to the carriers in the barriers since the hole density in the barriers gradually increases as a result of thermionic emission and ionization of acceptors and dominates the conductivity at high temperature as described above. Therefore, at higher temperatures, the scattering of holes will be mainly via LO phonon in the GaAs layers where the mobility is rapidly reduced to a very low value, $\mu_{H} \sim 198 \mathrm{~cm}^{-2} / \mathrm{V} \mathrm{s}$ at room temperature (RT), which is close to the reported value of the hole mobility in $p$-type GaAs epilayer with similar hole concentration. ${ }^{34,35}$ In order to show the different temperature regions, we used Matthiessen's rule to obtain the optical phonon scattering limited mobility $\mu_{\mathrm{LO}}$,

$$
\frac{1}{\mu_{\mathrm{LO}}}=\frac{1}{\mu_{T_{L}}}-\frac{1}{\mu_{0}},
$$

where $\mu_{T_{L}}=\mu_{H}$ is the total (measured) mobility. The low temperature mobility $\mu_{0}=3125 \mathrm{~cm}^{-2} / \mathrm{V} \mathrm{s}$ includes all the scattering mechanisms dominant at low temperatures (remote impurity, interface roughness, and acoustic phonon scattering). We then plotted the natural logarithm of $1 / \mu_{\mathrm{LO}}$ as a function of $1 / T_{L}$, as depicted in Fig. 4. It is evident from the figure that $1 / \mu_{\mathrm{LO}}$ has a $e^{\left(-\hbar \omega_{\mathrm{LO}} / k_{B} T\right)}$ dependence in two ranges of temperature. At temperatures between $T_{L}=65$ and $120 \mathrm{~K}$, the optical phonon energy $\hbar \omega_{\mathrm{LO}}$ has a value of around $25 \mathrm{meV}$, while at $T_{L}>120 \mathrm{~K}$, it is $37 \mathrm{meV}$. These values are close to the $\mathrm{LO}$ phonon energies of $29 \mathrm{meV}$ in GaInNAs and $36 \mathrm{meV}$ in GaAs. ${ }^{26}$ This observation confirms that the transport at temperatures below $120 \mathrm{~K}$ is dominated by holes in the QWs, while at $T_{L}>120 \mathrm{~K}$, it is increasingly dominated by carriers in the barrier layers. In Fig. 4, the gradually decreasing slope of the curve at temperatures below $65 \mathrm{~K}$ is indicative of the increasing involvement of low energy acoustic phonons in the momentum relaxation.

High speed $I-V$ measurements for conduction parallel to the $2 \mathrm{D}$ layers were carried out using several simple bars with lengths ranging from 50 to $100 \mu \mathrm{m}$. In order to reduce Joule heating, electric field pulses of $70 \mathrm{~ns}$ duration with a duty cycle less than $10^{-5} \mathrm{~s}$ were applied along the samples. I- $V$ characteristics were then recorded using a $500 \mathrm{MHz}$ oscilloscope. In Fig. 5, we plot the measured current as a function of the applied field at two different lattice temperatures $\left(T_{L}\right.$ $=13 \mathrm{~K}$ and $T_{L}=293 \mathrm{~K}$ ). At both temperatures, the current initially increases linearly with electric field at low fields (Ohmic region). It then deviates from linearity as expected from increased momentum scattering of hot holes with optical LO phonons. ${ }^{36}$ The most interesting behavior of the current-field characteristic is the tendency toward current saturation in a small range of electric fields $(F \sim 6 \mathrm{kV} / \mathrm{cm})$ at $T_{L}=13 \mathrm{~K}$. This region, which corresponds to a drift velocity $v_{d s} \approx 1 \times 10^{7} \mathrm{~cm} / \mathrm{s}$, is followed by a rapid increase in the current with increasing electric field. However, at $T_{L}$ $=293 \mathrm{~K}$, not only does the saturation occur at very high fields $(F \sim 19.3 \mathrm{kV} / \mathrm{cm})$ but also the current no longer increases with field above the saturation threshold. Measurements on several simple bars with different channel lengths exhibit the same behavior. We believe (and show below) that at the saturation threshold electric field $F \sim 6 \mathrm{kV} / \mathrm{cm}$ at $T_{L}$ $=13 \mathrm{~K}$, the nonequilibrium temperature of holes is high enough to cause a significant population of holes to transfer in real space via thermionic emission into the GaAs
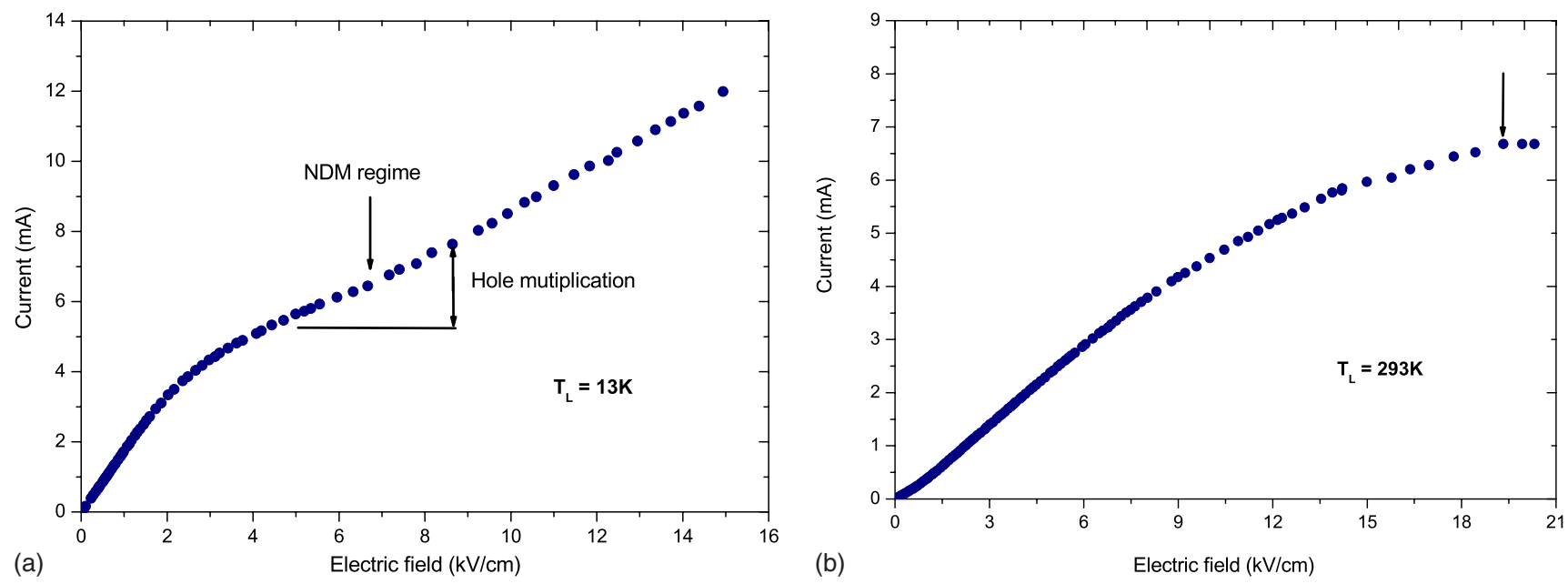

FIG. 5. (Color online) Measured current vs applied electric field at (a) $T_{L}=13 \mathrm{~K}$ and (b) $T_{L}=293 \mathrm{~K}$. 

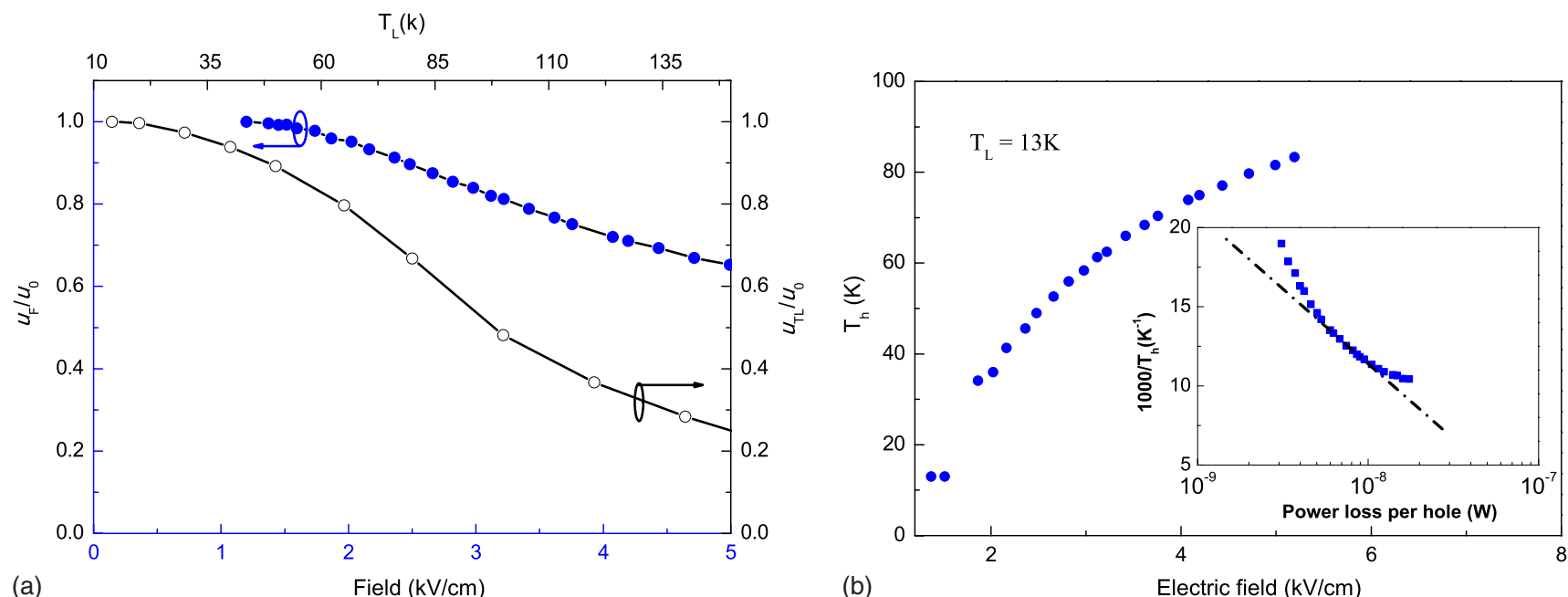

FIG. 6. (Color online) (a) Normalized mobilities as a function of applied electric field (lower horizontal axis) at $T_{L}=13 \mathrm{~K}$ and lattice temperature (upper horizontal axis). (b) Hole temperatures vs applied electric field measured at $T_{L}=13 \mathrm{~K}$. The inset shows inverse hole temperature vs power loss per hole and the broken line has a slope of $\hbar \omega_{\mathrm{LO}}=24.7 \mathrm{meV}$.

barriers. ${ }^{18,19}$ At low lattice temperature $\left(T_{L}=13 \mathrm{~K}\right)$ and low electric fields, the holes reside primarily in the GaInNAs potential wells in equilibrium, where they experience reduced local impurity scattering. This coupled with the lack of nitrogen related alloy scattering and low effective mass $\left(0.105 m_{0}\right)($ Ref. 17) implies that the hole mobility in the well is much higher than that in the Be-doped barrier $\left(\mu_{2}\right.$ $\sim 400 \mathrm{~cm}^{-2} / \mathrm{V}$ s in our calculation), where the hole effective mass is high and the local impurity scattering is very strong. As a result, current flow is essentially due to the hole transport in the well as we demonstrate above. At moderately high electric fields applied parallel to the layers, however, highmobility holes are rapidly heated up to energies well above their thermal distribution and transfer into the adjacent lowmobility GaAs layers. Therefore, the sample exhibits RST induced NDM at electric fields around $7 \mathrm{kV} / \mathrm{cm}$ as expected. However, at RT, the current is due primarily to the carriers in the low-mobility GaAs layer as we showed above. Therefore, at RT there is no RST and the drift velocity saturates at very high fields at which necessary input power is achieved to reach the momentum balance in both polar and nonpolar phonon interactions.

In order to evaluate the nonequilibrium hole temperatures as a function of applied electric field and the energy loss rate, we used the mobility mapping technique. In this technique, the hole mobility at $T_{L}=13 \mathrm{~K}$ is obtained as a function of applied field $\mu_{F}$ from measured $I-V$ characteristics by assuming that the hole density is constant within the range of applied electric field $F<6 \mathrm{kV} / \mathrm{cm}$. The normalized mobility is then obtained from the Ohmic and nonlinear resistances via

$$
\begin{aligned}
& R_{0}=\left(\frac{V}{I}\right)_{F \rightarrow 0}=\left(e p_{0} \mu_{0}\right)^{-1} \times \frac{l}{w \times t}, \\
& R_{F}=\left(\frac{V}{I}\right)_{F}=\left(e p_{F} \mu_{F}\right)^{-1} \times \frac{l}{w \times t},
\end{aligned}
$$

where $R_{0}, R_{F}, p_{0}, p_{F}, \mu_{0}$, and $\mu_{F}$ are the Ohmic resistance at low field $(F \rightarrow 0)$, the field dependent resistance, the 3D car- rier density at $T_{L}=13 \mathrm{~K}$ when $F \rightarrow 0$, the field dependent 3D carrier density, the carrier mobility at $T_{L}=13 \mathrm{~K}$ when $F$ $\rightarrow 0$, and the field dependent carrier mobility, respectively. $l$, $w$, and $t$ are the sample length, width, and thickness of the conduction channel $(\mathrm{QW})$, respectively, and $e$ is the electronic charge. Therefore, the field dependent mobility normalized with respect to the Ohmic mobility is

$$
\frac{\mu_{F}}{\mu_{0}}=\frac{p_{0} R_{0}}{p_{F} R_{F}}=\frac{R_{0}}{R_{F}}
$$

Here it is assumed that the carrier density in the QW is independent of the applied field, i.e., $p_{0}=p_{F}$.

The measured normalized mobility $\mu_{F} / \mu_{0}$ is plotted against electric field in Fig. 6(a). The low-field mobility at a given lattice temperature, $\mu_{\mathrm{TL}}$, is also normalized with respect to $\mu_{0}$, i.e., $\mu_{\mathrm{TL}} / \mu_{0}$ is also plotted against the lattice temperature in the same figure. Assuming that increasing the lattice temperature and hole temperature have the same effect on the momentum relaxation in the $\mathrm{LO}$ phonon regime, $, 36,37$ the nonequilibrium hole temperature as a function of electric field can then be extracted directly by comparing the electric field dependence of $\mu_{F} / \mu_{0}$ and temperature dependence of $\mu_{\mathrm{TL}} / \mu_{0}$. The nonequilibrium temperatures of holes obtained by this method are plotted in Fig. 6(b) as a function of electric field. It is worth noting that the hole temperature appears to increase very little at $F>5 \mathrm{kV} / \mathrm{cm}$. This does not mean that the hole temperature saturates because above the RST threshold, the carrier density in the well is no longer constant, and hence, the mobility mapping technique is no longer valid.

In the steady state, the power input per hole is equal to that lost to the lattice through scattering processes; thus the rate of power loss per hole can be obtained as a function of hole temperature, as shown in the inset of Fig. 6(b). The broken line drawn through the experimental points is the best fit from the energy relaxation rate involving only LO phonons in the form ${ }^{38}$ 


$$
p=e \mu_{F} F^{2}=\frac{\hbar \omega_{\mathrm{LO}}}{\left\langle\tau_{h}\right\rangle} \exp \left(-\hbar \omega_{\mathrm{LO}} / k_{B} T_{h}\right),
$$

where $p, \hbar \omega_{\mathrm{LO}},\left\langle\tau_{h}\right\rangle, T_{h}$, and $k_{B}$ are the power loss per hole, the LO phonon energy, the characteristic time constant for the polar interaction, the hole temperature, and Boltzmann's constant, respectively. It has a slope of about $24.7 \mathrm{meV}$ which agrees well with the LO phonon energy obtained above using Matthiessen's rule.

\section{THEORY AND THE DISCUSSION OF RESULTS}

In order to simulate the observed current-voltage characteristics at low lattice temperature, we use a simplified analytical model taking into account the RST of holes from high-mobility wells into low-mobility barriers. Due to the wide barriers, the tunneling between the wells is negligibly small. Therefore, the holes confined in the wells are quasi-2D and have a spatially uniform nonequilibrium temperature $T_{1}$, while the holes in the GaAs barriers are assumed to be bulklike and also have a spatially uniform temperature of $T_{2}<T_{1}$. Since the intrasubband scattering processes are expected to occur much more frequently than the transfer scattering from a bound state to an unbound state above the barrier, it is possible to treat the subbands as two separate subsystems, ${ }^{39}$ each of which has its own drift velocity $V_{d i}$, hole temperature $T_{i}$, and chemical potential $\zeta_{i}$ (where $i=1,2$ indexes the well and barrier regions, respectively). In order to obtain the analytical solution, we introduce the following assumptions concerning the holes.

(i) The effect of size quantization and the compressive strain in a narrow well lead to a large splitting of the heavy-hole subbands and the light-hole subbands, and hence largely reduce nonparabolicity and anisotropy of the highest subband $\left(\mathrm{HH}_{1}\right)$. This allows us to consider holes confined in the $\mathrm{HH}_{1}$ subband. For simplicity, we restrict our attention to the holes in a single parabolic band, which is acceptable because the hole temperatures considered here are not high enough to deviate from the parabolicity of the energy spectrum.

(ii) At low lattice temperatures, there is a considerable freeze-out of carriers in the GaAs barrier at thermal equilibrium. We therefore assume that all free holes in the barrier are from holes out of the well via thermionic emission.

(iii) The band bending caused by the ionized negative acceptors is estimated to be lower than the center of the barrier by about $30 \mathrm{meV}$ at thermal equilibrium, which is much lower than the valence band discontinuity $\sim 120 \mathrm{meV}$ (provided it is not so strong that the potential wells in the barriers give rise to strongly confined states). Thus we adopt a flatband approximation for simplicity.

(iv) The effect of nonequilibrium phonons on both the energy and momentum relaxation is ignored due to the high effective density of states and reduced lifetime of phonons. ${ }^{40}$

The drift velocity versus electric field curve for each subsystem can then be deduced from the energy and momentum balance equations under steady-state condition. For simplicity, we assume the holes in both subsystems interacting solely with polar optical phonon scattering for energy relaxation. We then have a set of equations. In the GaInNAs QW, the energy relaxation rate $f_{P O}$ and the momentum relaxation $\mu_{P O}$ for the $2 \mathrm{D}$ hole-interface $\mathrm{LO}$ phonon interaction can be written as ${ }^{41}$

$$
\begin{aligned}
e F V_{d 1} & =\mu_{1}\left(T_{1}\right) F^{2}=f_{P O}\left(T_{1}\right) \\
& =\frac{\hbar \omega_{1}}{\tau_{0}}\left(\frac{E_{L}}{\hbar \omega_{1}}\right)^{1 / 2}\left\{\left[n_{0}\left(\omega_{1}\right)+1\right] \exp \left(-\frac{\hbar \omega_{1}}{k_{B} T_{1}}\right)-n_{0}\left(\omega_{1}\right)\right\}
\end{aligned}
$$

and

$$
\frac{1}{\mu_{P O}\left(T_{1}\right)}=\frac{m_{1}{ }^{*}\left(\hbar \omega_{1} E_{L}\right)^{1 / 2}}{q \tau_{0} k_{B} T_{1}}\left[n_{0}\left(\omega_{1}\right)+1\right] \exp \left(-\hbar \omega_{1} / k_{B} T_{1}\right),
$$

respectively. Here, $\hbar \omega_{1}$ is the LO phonon energy in the well, $n_{0}\left(\omega_{1}\right)=1 /\left[\exp \left(\hbar \omega_{1} / k_{B} T_{0}\right)-1\right]$ is the equilibrium phonon occupation number at lattice temperature $T_{0}, E_{L}=\hbar^{2} \pi^{2}$ / $2 m_{1}{ }^{*} L_{z}{ }^{2}$ is the energy shift of the lowest subband in an infinitely deep well of width $L_{z}$, and $m_{1}{ }^{*}, q$, and $k_{B}$ are the effective hole mass in the well, the positive electronic charge, and Boltzmann's constant, respectively. $\tau_{0}$ is a characteristic time constant for the polar interaction given by

$$
\tau_{0}=\frac{q^{2} \omega_{1}}{2 \pi \hbar}\left(\frac{m_{1}^{*}}{2 \hbar \omega_{1}}\right)^{1 / 2}\left(\frac{1}{\varepsilon_{\infty 1}}-\frac{1}{\varepsilon_{s 1}}\right),
$$

where $\varepsilon_{\infty}$ and $\varepsilon_{s}$ are the high-frequency and static permittivities, respectively. For momentum relaxation, in addition to the LO phonon scattering, we introduce a further elastic scattering in power law form with an arbitrary temperature dependence $r_{1}$ to correct the low-field mobility,

$$
\mu_{e}\left(T_{1}\right)=\mu_{e}\left(T_{0}\right)\left(\frac{T_{0}}{T_{1}}\right)^{r_{1}},
$$

where $r_{1}$ can be determined from the best fit to the experimental data and the value of $\mu_{e}\left(T_{0}\right)$ can be calculated in accordance with the low-field mobility $\mu_{0}$ using Matthiessen's rule,

$$
\frac{1}{\mu_{e}\left(T_{0}\right)}=\left(\frac{1}{\mu_{0}}-\frac{1}{\mu_{P O}\left(T_{0}\right)}\right),
$$

where $\mu_{P O}\left(T_{0}\right)$ can be obtained from Eq. (7) in the limit $T_{1}$ $\rightarrow T_{0}$,

$$
\frac{1}{\mu_{P O}\left(T_{0}\right)}=\frac{m_{1}^{*}\left(\hbar \omega_{1} E_{L}\right)^{1 / 2}}{q \tau_{0} k_{B} T_{0}} n_{0}\left(\omega_{1}\right) .
$$

We then have the total momentum relaxation rate for the 2D holes in the well,

$$
\frac{1}{\mu_{1}\left(T_{1}\right)}=\frac{1}{\mu_{P O}\left(T_{1}\right)}+\left(\frac{1}{\mu_{0}}-\frac{1}{\mu_{P O}\left(T_{0}\right)}\right)\left(\frac{T_{1}}{T_{0}}\right)^{r_{1}} .
$$


Holes in the GaAs barrier scatter primarily from ionized impurities and bulk LO phonons. The energy relaxation rate per hole for the 3D hole-bulk LO phonon interaction is given by $^{42}$

$$
\begin{aligned}
e F V_{d 2}= & \mu_{2}\left(T_{2}\right) F^{2}=f_{p o}\left(T_{2}\right) \\
= & \left(\frac{2}{\pi}\right)^{1 / 2} \frac{\hbar \omega_{2}}{\tau_{0}}\left\{\left[n_{0}\left(\omega_{2}\right)+1\right] \exp \left(-\hbar \omega_{2} / k_{B} T_{2}\right)-n_{0}\left(\omega_{2}\right)\right\} \\
& \times\left(\frac{\hbar \omega_{2}}{2 k_{B} T_{2}}\right)^{1 / 2} \exp \left(\hbar \omega_{2} / 2 k_{B} T_{2}\right) K_{0}\left(\hbar \omega_{2} / 2 k_{B} T_{2}\right),
\end{aligned}
$$

where $\hbar \omega_{2}$ is the phonon energy in the GaAs barrier and $K_{0}(x)$ is the modified Bessel function. $\tau_{0}$ and $n_{0}\left(\omega_{2}\right)$ have the same meaning, as defined in Eq. (6). The momentum relaxation for 3D holes via the interaction with bulk LO phonons can be found using ${ }^{41}$

$$
\begin{aligned}
\frac{1}{\mu_{P O}\left(T_{2}\right)}= & \frac{m_{2}^{*}}{3 \pi^{1 / 2} q \tau_{0}}\left(\frac{\hbar \omega_{2}}{k_{B} T_{2}}\right)^{3 / 2}\left\{\left\{\left[n_{0}\left(\omega_{2}\right)+1\right]\right.\right. \\
& \left.\times \exp \left(-\hbar \omega_{2} / k_{B} T_{2}\right)+n_{0}\left(\omega_{2}\right)\right\} \\
& \times \exp \left(\hbar \omega_{2} / 2 k_{B} T_{2}\right) K_{1}\left(\hbar \omega_{2} / 2 k_{B} T_{2}\right) \\
& +\left\{\left[n_{0}\left(\omega_{2}\right)+1\right] \exp \left(-\hbar \omega_{2} / k_{B} T_{2}\right)-n_{0}\left(\omega_{2}\right)\right\} \\
& \left.\times \exp \left(\hbar \omega_{2} / 2 k_{B} T_{2}\right) K_{0}\left(\hbar \omega_{2} / 2 k_{B} T_{2}\right)\right\} .
\end{aligned}
$$

In addition to the scattering from LO optical phonons, the dominant contribution to the momentum relaxation of $3 \mathrm{D}$ holes is from ionized local impurities and can be presented in the following form at low electric field: ${ }^{35}$

$$
\mu_{\mathrm{im}}^{\mathrm{eff}}\left(T_{0}\right)=W \frac{r^{1 / 2}+r^{3 / 2}}{1+r^{3 / 2}} \mu_{\mathrm{im}}^{s}\left(T_{0}\right),
$$

where $W(1 \leq W \leq 2)$ is a factor accounting for the $p$-like symmetry of the hole wave functions, $r=m_{\mathrm{hh}} / m_{\mathrm{lh}}$ is the ratio of effective mass of heavy hole $\left(0.62 m_{0}\right)$ (Ref. 35) and light hole $\left(0.074 m_{0}\right)$ (Ref. 35), and $\mu_{\mathrm{im}}^{s}$ can be calculated using the Conwell-Weisskopf approach and is given by ${ }^{35}$

$$
\begin{aligned}
\mu_{\mathrm{im}}^{s}\left(T_{0}\right)= & \frac{3.68 \times 10^{20}}{N_{i}}\left(\frac{\varepsilon}{16}\right)^{2}\left(\frac{T_{0}}{100}\right)^{1.5} \\
& \times \frac{1}{\left(m_{2}{ }^{*} / m_{0}\right)^{1 / 2} \log \left(1+\beta_{\mathrm{cw}}^{2}\right)},
\end{aligned}
$$

where $\beta_{\mathrm{cw}}=(\varepsilon / 16)\left(T_{0} / 100\right)\left(2.35 \times 10^{19} / N_{i}\right)^{1 / 3}, \varepsilon$ is the static dielectric constant, and $N_{i}$ is the ionized impurity concentration. Using the power law, the energy dependence of charged impurity scattering can be obtained from $\mu_{\mathrm{im}}^{\text {eff }}\left(T_{2}\right)=\mu_{\mathrm{im}}^{\text {eff }}\left(T_{0}\right)$ $\times\left(T_{2} / T_{0}\right) r_{2}$ (where $r_{2}$ can be determined from the best fit to the experimental data). The total momentum relaxation in the barrier is then given by

$$
\frac{1}{\mu_{2}\left(T_{2}\right)}=\frac{1}{\mu_{P O}\left(T_{2}\right)}+\frac{1}{\mu_{\mathrm{im}}^{\text {eff }}\left(T_{2}\right)} .
$$

In order to determine the quasi-Fermi levels and carrier density in each subsystem, we adopted an additional condition: the balance of the thermionic currents in both directions at the interface in the steady state. The thermionic currents flowing from the GaInNAs well to the GaAs barrier $j_{1-2}$, and
TABLE I. Values of material constants used in the calculation for sample 1931.

\begin{tabular}{lcc}
\hline \hline Parameter & GaInNAs & GaAs \\
\hline High frequency dielectric constant & $\varepsilon_{\infty 1}=11.11$ & $\varepsilon_{\infty 2}=10.6$ \\
Low frequency dielectric constant & $\varepsilon_{S 1}=13.575$ & $\varepsilon_{S 2}=12.9$ \\
Polar optical phonon energy & $\hbar \omega_{1}=25 \mathrm{meV}$ & $\hbar \omega_{2}=36 \mathrm{meV}$ \\
Hole effective mass & & \\
$\left(m_{0}=9.1095 \times 10^{-31} \mathrm{~kg}\right)$ & $m_{1}{ }^{*}=0.105 m_{0}$ & $m_{2}{ }^{*}=0.62 m_{0}$ \\
Quantum well/barrier width & $L_{z}=7 \mathrm{~nm}$ & $L_{2}=20 \mathrm{~nm}$ \\
Power parameter & $r_{1}=0.167$ & $r_{2}=0.5$ \\
\hline \hline
\end{tabular}

from the GaAs layer to the well $j_{2-1}$, can be calculated using Bethe's theory and are given by ${ }^{4}$

$$
\begin{aligned}
& J_{1-2}=q p_{1}\left(\frac{k_{B} T_{1}}{2 \pi m_{1}{ }^{*}}\right)^{1 / 2} \exp \left(-\frac{\Delta E_{V}}{k_{B} T_{1}}\right), \\
& J_{2-1}=q p_{2}\left(\frac{k_{B} T_{2}}{2 \pi m_{2}{ }^{*}}\right)^{1 / 2} \exp \left(-\frac{\phi_{b}}{k_{B} T_{2}}\right),
\end{aligned}
$$

where $p_{1}$ and $p_{2}$ are the 3D hole densities in GaInNAs QW and GaAs barrier, respectively, $\Delta E_{V} \approx 110 \mathrm{meV}$ is the hole confinement, and $\phi_{b} \approx 30 \mathrm{meV}$ is the space-charge potential barrier. Under steady-state conditions, we then have $J_{1-2}$ $=J_{2-1}$. Since the hole numbers in the two subbands are related to each other and the total number of holes is conserved, we then have

$$
p_{0} L_{z}=p_{1} L_{z}+p_{2} L_{2},
$$

where $p_{0}$ is the 3D hole density in the well at low field, and $L_{2}$ is the width of the barrier. Finally, the field dependent current of the GaInNAs/GaAs QW-barrier system can then be calculated by

$$
I=I_{1}+I_{2}=q p_{1} V_{d 1} L_{z} w+q p_{2} V_{d 2} L_{2} w,
$$

where $w=2.8 \times 10^{-3} \mathrm{~cm}$ is the width of the simple bar.

The balance equations have been solved numerically for sample 1931 at $T_{0}=13 \mathrm{~K}$ taking the parameters given in Table I. The resulting drift velocities versus electric field are shown in Fig. 7. The drift velocity of 3D holes is much lower than that of 2D holes due to the strong local ionized impurity scattering, which dominates the momentum relaxation at electric fields up to approximately $3.2 \mathrm{kV} / \mathrm{cm}$, where the LO phonon scattering becomes more important. On the other hand, the high drift velocity of the 2D holes is accompanied by a rapid increase in their temperature with the rising electric field. As a result, the LO phonon scattering of 2D holes becomes dominant at much lower electric field (approximately $0.8 \mathrm{kV} / \mathrm{cm}$ ). At fields higher than approximately $6 \mathrm{kV} / \mathrm{cm}$, the $2 \mathrm{D}$ carriers in the well are considerably heated which allows a significant fraction of holes to be scattered into the 3D subband and the barrier layer starts to be populated, as shown in Fig. 8. As the electric field is further increased, more carriers are transferred into the barrier layer and the inversion of the carrier population is reached at fields above approximately $8.4 \mathrm{kV} / \mathrm{cm}$.

In Fig. 9, the calculated total currents for the GaInNAs/ GaAs system including the contributions from both the 


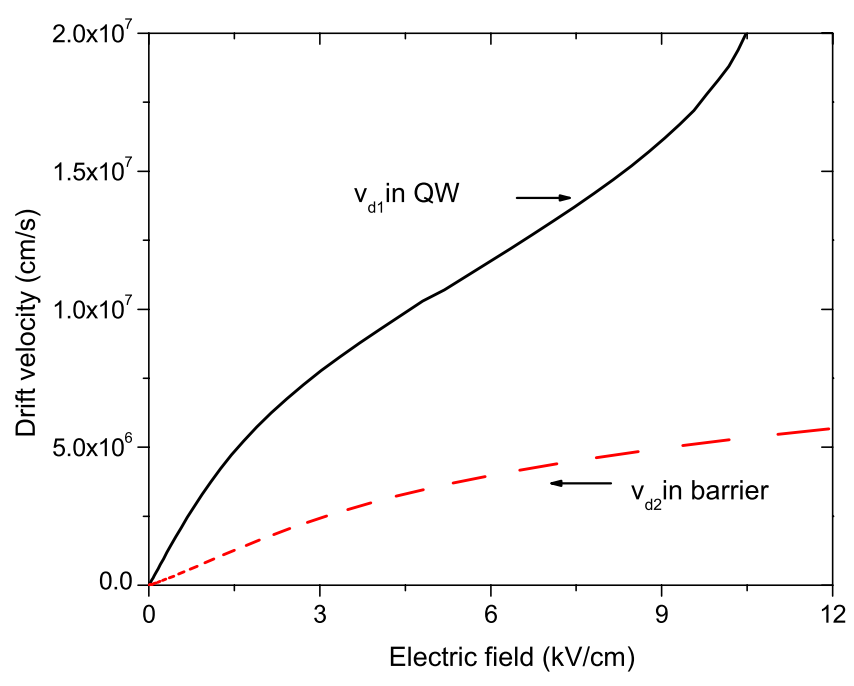

FIG. 7. (Color online) Calculated drift velocity of the 2D holes in the well (full line) and the 3D holes in the barrier (dashed line) as a function of applied field.

GaInNAs well and the GaAs barrier are plotted as a function of electric field. Also shown in the figure are the experimental results, represented by solid diamonds. It is clearly seen from the figure that for fields up to approximately $6 \mathrm{kV} / \mathrm{cm}$, the overall current flow is essentially due to the $2 \mathrm{D}$ hole transport in the GaInNAs well. However, as the field further increases, the number of holes in the well steadily reduces resulting in the contribution from $2 \mathrm{D}$ holes to the overall current falling off. On the other hand, the 3D hole part of the current increases with rising electric field, but it cannot compensate for the decrease in the 2D hole part of the current due to the low drift velocity in the GaAs barrier. This leads to NDM for fields above $\sim 7 \mathrm{kV} / \mathrm{cm}$. Comparison of calculated with measured currents indicates that the experimental data only agree very well with the calculated results for fields below approximately $6 \mathrm{kV} / \mathrm{cm}$, for fields above it; however, the values of measured current are higher than the calculated ones. Furthermore, the measured current exhibits NDM characteristic at fields around $7 \mathrm{kV} / \mathrm{cm}$, but it sharply increases as

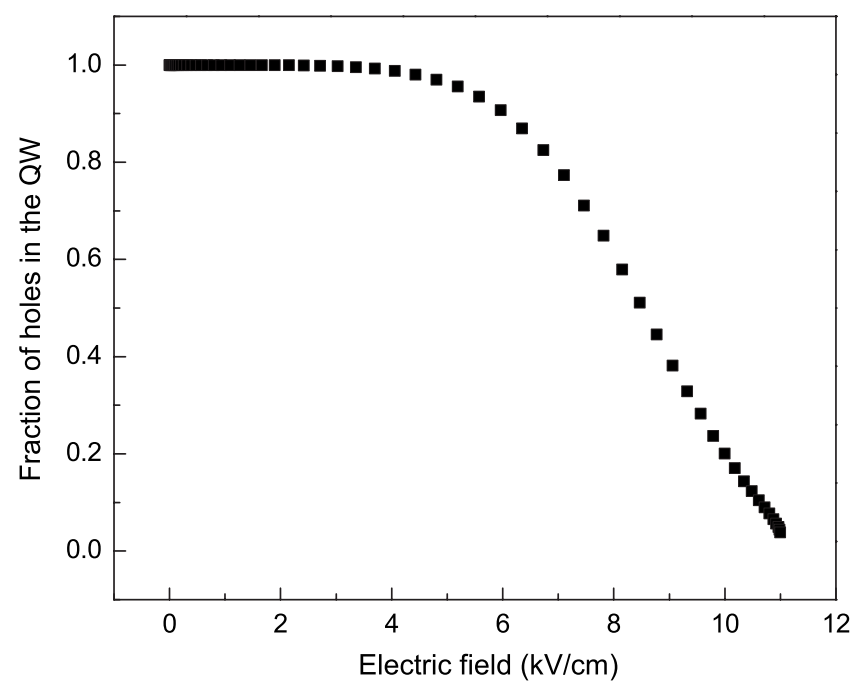

FIG. 8. Calculated fraction of the holes in the GaInNAs well as a function of electric field.

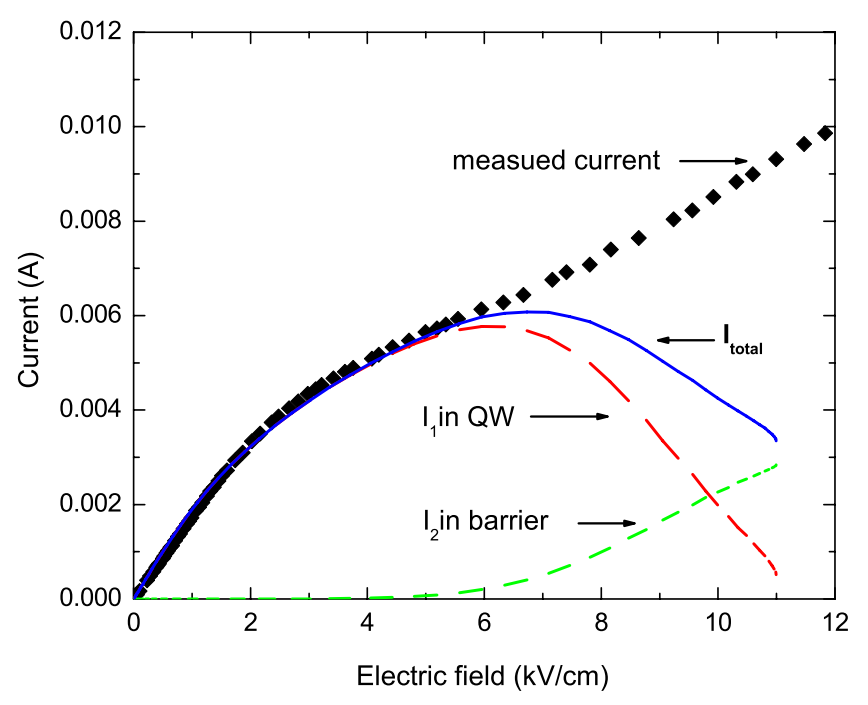

FIG. 9. (Color online) Calculated current vs applied field at $T_{0}=13 \mathrm{~K}$ (full line). Two dashed lines show the contributions from the 2D subband (long dashed line) and the 3D subband (short dashed line) to the current. The solid diamonds represent the experimental data.

the electric field further increases. These interesting observations can be well explained in terms of hole multiplication. At electric fields above about $6 \mathrm{kV} / \mathrm{cm}$, the transferred holes from the GaInNAs well can impact ionize the acceptors giving rise to the increase in the current in the GaAs barrier. Since at high electric fields the current is dominated by the 3D hole transport in the barrier (Fig. 8), we calculated the 3D hole part of the current by assuming that all the acceptors are ionized. However, the calculated current is much lower than the experimental data. This strongly suggests the presence of an additional mechanism leading to a sharp increase in the current. As it is well know, in the N-NDM regime, homogeneous fields are always unstable and the high-field domain associated with RST of holes can be formed in the well. In the high-field domain region, band-to-band impurity ionization may occur resulting in the creation of holes having high mobility in the well. Therefore, we believe that the occurrence of impact ionization in both the barrier and well leads to the observed current surge. The direct experimental proofs confirming the impact ionization are now under investigation and the results are expected to be published in the near future.

\section{SUMMARY}

In conclusion, we have studied the energy and momentum relaxation of hot holes in the $\mathrm{Ga}_{x} \mathrm{In}_{1-x} \mathrm{~N}_{y} \mathrm{As}_{1-y} / \mathrm{GaAs}$ modulation doped quantum-well structures. The results are compared with a simplified theory based on the energy and momentum balance method including the effect of RST of hot holes in the GaInNAs well. It has been shown that the RST of hot holes only occurs at low temperature and the observed complicated hole transport behavior in the GaInNAs/GaAs system can be well understood in terms of the RST effect and hole multiplication induced by impurity breakdown in the GaAs barrier and impact ionization in the high-field domain regime traveling along the applied field direction. This unusual high-field transport behavior of 2D 
holes in $p$-type GaInNAs material is expected to open up a wide range of possible applications. Shallow impurity breakdown in the Be-doped GaAs layer allows the direct transition from the valence band to the acceptor level, which may result in microwave radiation in the terahertz frequency range. ${ }^{44}$ Furthermore, excess carriers created due to fieldinduced impact ionization inside the traveling domain may result in light emission via a number of radiative recombination mechanisms such as interband recombination of the impact ionized electron-hole pairs and bremsstrahlung. These mechanisms have been exploited for many devices such as metal-semiconductor field effect transistors ${ }^{45}$ and Gunn laser, ${ }^{46}$ based on the GaAs material system. In addition, the effect of RST can lead to intrinsic self-generated oscillations in the range $20-80 \mathrm{GHz},{ }^{22}$ which is of considerable interest for applications as a simple way of generating highfrequency microwave power based on the GaInNAs material system.

\section{ACKNOWLEDGMENTS}

We are grateful to the University of Essex RPF for funding the project and to Dr. C. Fontaine for growing the samples.

${ }^{1}$ J. S. Harris, Semicond. Sci. Technol. 17, 880 (2002).

${ }^{2}$ B. Borchert, A. Y. Egorov, S. Illek, M. Komainda, and H. Riechert, Electron. Lett. 35, 2204 (1999).

${ }^{3}$ P. C. Chang, A. G. Baca, N. Y. Li, P. R. Sharp, H. Q. Hou, J. R. Laroche, and F. Ren, Appl. Phys. Lett. 76, 2788 (2000).

${ }^{4}$ D. J. Friedman, J. F. Geisz, S. R. Kurtz, and J. M. Olson, J. Cryst. Growth 195, 409 (1998).

${ }^{5}$ D. B. Jackrel, S. R. Bank, H. B. Yuen, M. A. Wistey, J. S. Harris, A. J. Ptak, S. W. Johnston, D. J. Friedman, and S. R. Kurtz, J. Appl. Phys. 101, 114916 (2007).

${ }^{6}$ M. Weyers, M. Sato, and H. Ando, Jpn. J. Appl. Phys., Part 2 31, L853 (1992).

${ }^{7}$ M. Kondow, K. Uomi, K. Hosomi, and T. Mozume, Jpn. J. Appl. Phys., Part 2 33, L1056 (1994).

${ }^{8}$ W. Li, M. Pessa, J. Toivonen, and H. Lipsanen, Phys. Rev. B 64, 113308 (2001).

${ }^{9}$ R. Mouillet, L. A. de Vaulchier, E. Deleporte, Y. Guldner, L. Travers, and J. C. Harmand, Solid State Commun. 126, 333 (2003).

${ }^{10}$ J. Teubert, P. J. Klar, W. Heimbrodt, K. Volz, and W. Stolz, IEE Proc.: Optoelectron. 151, 357 (2004).

${ }^{11}$ C. Skierbiszewski, P. Wisniewski, T. Suski, W. Walukiewicz, W. Shan, J. W. Ager, E. E. Haller, J. F. Geisz, D. J. Freidman, J. M. Olson, and S. R. Kurtz, Phys. Status Solidi B 216, 135 (1999).

${ }^{12}$ M. P. Vaughan and B. K. Ridley, Phys. Rev. B 75, 195205 (2007).

${ }^{13}$ S. Fahy and E. P. O'Reilly, Physica E (Amsterdam) 21, 881 (2004).

${ }^{14}$ S. Fahy and E. P. O'Reilly, Appl. Phys. Lett. 83, 3731 (2003).

${ }^{15}$ S. Fahy, A. Lindsay, H. Ouerdane, and E. P. O’Reilly, Phys. Rev. B 74 035203 (2006).

${ }^{16}$ Y. Sun, A. Erol, M. Yilmaz, M. C. Arikan, B. Ulug, A. Ulug, N. Balkan, M. Sopanen, O. Reentilä, M. Mattila, C. Fontaine, and A. Arnoult, Opt.
Quantum Electron. 40, 467 (2008).

${ }^{17}$ Y. Sun, N. Balkan, M. Aslan, S. B. Lisesivdin, H. Carrere, and X. Marie, J. Phys.: Condens. Matter 21, 174210 (2009).

${ }^{18}$ An analytical model was developed to analyze the observed transition energy between the conduction and valence subbands for GaInNAs/GaAs QWs. The model was derived using the band anticrossing approach accounting for finite QW confinement and strain effect, and treating the valence band separately. In the model, all energy levels are aligned to VB of GaAs using the model-solid theory. The results can be well fitted to the transition energies obtained from photoluminescence data and give large conduction band discontinuity $(\sim 482 \mathrm{meV})$ and much smaller valence band discontinuity $(\sim 120 \mathrm{meV})$. The hh1 level is calculated to be 12 meV below VB edge of GaInNAs, which gives the hole confinement about $110 \mathrm{meV}$.

${ }^{19}$ K. Hess, H. Morkoc, H. Shichijo, and B. C. Streetman, Appl. Phys. Lett. 35, 469 (1979).

${ }^{20}$ K. Hess, J. Phys. Colloq. 42, C7-3 (1981).

${ }^{21}$ R. Döttling and E. Schöll, Phys. Rev. B 45, 1935 (1992).

${ }^{22}$ E. Schöll and K. Aoki, Appl. Phys. Lett. 58, 1277 (1991).

${ }^{23}$ D. C. Look, D. C. Walters, M. O. Manasreh, J. R. Sizelove, C. E. Stutz, and K. R. Evans, Phys. Rev. B 42, 3578 (1990).

${ }^{24}$ P. W. Yu, G. D. Robinsob, J. R. Sizelove, and C. E. Stutz, Phys. Rev. B 49, 4689 (1994).

${ }^{25}$ M. Shinohara, J. Appl. Phys. 61, 365 (1987).

${ }^{26}$ R. J. Potter and N. Balkan, J. Phys.: Condens. Matter 16, S3387 (2004).

${ }^{27}$ A. Polimeni, M. Capizzi, M. Geddo, M. Fischer, M. Reinhardt, and A. Forchel, Appl. Phys. Lett. 77, 2870 (2000).

${ }^{28}$ Y. P. Varshni, Physica (Utrecht) 34, 149 (1967).

${ }^{29}$ Y. Sun, M. Vaughan, A. Agarwal, M. Yilmaz, B. Ulug, A. Ulug, N. Balkan, M. Sopanen, O. Reentilä, M. Mattila, C. Fontaine, and A. Arnoult, Phys. Rev. B 75, 205316 (2007).

${ }^{30}$ M. J. Kane, N. Apsley, D. A. Anderson, L. L. Taylor, and T. Kerr, J. Phys. C 18, 5629 (1985)

${ }^{31}$ P. J. van Hall, T. Klaver, and J. H. Wolter, Semicond. Sci. Technol. 3, 120 (1988).

${ }^{32}$ A. Gold, Phys. Rev. B 35, 723 (1987).

${ }^{33}$ K. Greipel and U. Rössler, Semicond. Sci. Technol. 7, 487 (1992).

${ }^{34}$ F. E. Rosztoczy, F. Ermainis, I. Hayashi, and B. Schwartz, J. Appl. Phys. 41, 264 (1970).

${ }^{35}$ D. J. Wiley, Semiconductors and Semimetals (Academic, New York, 1975), Vol. 10

${ }^{36}$ J. Shah, A. Pinczuk, H. L. Stormer, A. C. Gossard, and W. Wiegmann, Appl. Phys. Lett. 44, 322 (1984).

${ }^{37}$ N. Balkan, R. Gupta, M. E. Danies, B. K. Ridley, and M. Emeny, Semicond. Sci. Technol. 5, 986 (1990).

${ }^{38}$ N. Balkan, B. K. Ridley, M. Emeny, and I. Goodridge, Semicond. Sci. Technol. 4, 852 (1989).

${ }^{39}$ V. Kubrak and P. Kleinert, J. Phys.: Condens. Matter 10, 7391 (1998).

${ }^{40}$ B. K. Ridley, Rep. Prog. Phys. 54, 169 (1991).

${ }^{41}$ B. K. Ridley, Semicond. Sci. Technol. 4, 1142 (1989).

${ }^{42}$ R. Stratton, J. Phys. Soc. Jpn. 17, 590 (1962).

${ }^{43}$ S. M. Sze, Physics of Semiconductor Devices, 2nd ed. (Wiley, New York, 1981).

${ }^{44}$ L. E. Vorob'ev, D. A. Firsov, V. A. Shalygin, V. Yu. Panevin, A. N. Sofronov, D. V. Tsoi, A. Yu. Egorov, A. G. Gladyshev, and O. V. Bondarenko, Tech. Phys. Lett. 32, 384 (2006).

${ }^{45}$ N. Balkan, B. K. Ridley, and A. J. Vickers, Negative Differential Resistance and Instabilities in 2-D Semiconductors (Plenum, New York, 1992).

${ }^{46}$ S. Chung and N. Balkan, Appl. Phys. Lett. 86, 211111 (2005). 